Port Acadie

Revue interdisciplinaire en études acadiennes

An Interdisciplinary Review in Acadian Studies

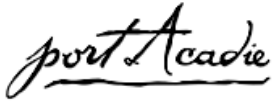

\title{
Aperçus de quelques vieux cimetières
}

\section{Sally Ross}

Numéro 10-11-12, automne 2006, printemps-automne 2007

Le patrimoine religieux de la Nouvelle-Écosse : signes et paradoxes en Acadie

URI : https://id.erudit.org/iderudit/018640ar

DOI : https://doi.org/10.7202/018640ar

Aller au sommaire du numéro

Éditeur(s)

Université Sainte-Anne

ISSN

1498-7651 (imprimé)

1916-7334 (numérique)

Découvrir la revue

Citer cet article

Ross, S. (2006). Aperçus de quelques vieux cimetières. Port Acadie, (10-11-12), 201-221. https://doi.org/10.7202/018640ar

\section{Résumé de l'article}

En 2003, l'auteur a visité les cimetières acadiens établis en Nouvelle-Écosse après la déportation. Le but de ce projet était de constituer une documentation des monuments funéraires d'intérêt historique, culturel et linguistique. À la lumière de ses recherches dans 61 sites, la chercheuse examine ici quatre cimetières paroissiaux emblématiques, dont deux à l'île du Cap-Breton (les vieux cimetières de Chéticamp et de Notre-Dame-de-l'Assomption) et deux en Nouvelle-Écosse péninsulaire (le cimetière Saint-Pierre de Pubnico-Ouest et celui de Sainte-Marie). En les situant d'abord dans un contexte historique, elle examine de près quelques-unes des pierres particulièrement intéressantes. 
DeuXIÈME PARTIE : L'ÉtAT DES LIEUX DE CULTE

LIEUX DE CULTE DU CAP-BRETON

\section{Aperçus de quelques vieux cimetières}

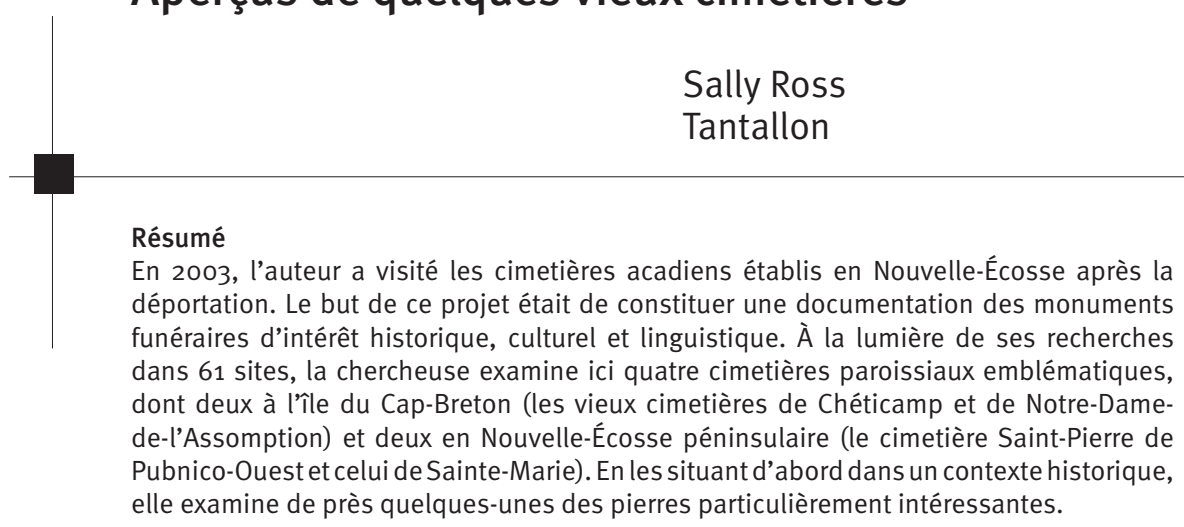

Le cimetière est avant tout un lieu sacré, mais il est aussi "la mémoire du temps $»^{1}$ et le témoin des générations passées. En 2003, j'ai décidé d'entreprendre une étude des cimetières, un aspect peu connu du patrimoine acadien qui m'intéresse depuis longtemps. En examinant les étapes de la vie, plusieurs folkloristes acadiens ont parlé des coutumes associées à la mort, mais en général leurs descriptions s'arrêtent avec l'enterrement. Ils parlent rarement des cimetières ou des pierres tombales. Plusieurs paroisses ont fait un relevé des inscriptions sur les pierres dans leur cimetière, mais les renseignements recueillis sont destinés surtout aux généalogistes. Mon intérêt se porte plutôt sur la culture matérielle et l'évolution du cimetière en tant que lieu de mémoire visible et invisible. Je voulais mener des recherches sur le terrain qui me permettraient de faire des analyses et des comparaisons. J'ai commencé donc par une tournée systématique de tous les cimetières acadiens de la Nouvelle-Écosse établis après la déportation afin de documenter les monuments funéraires d'intérêt historique, culturel et linguistique. J'ai invité Deborah Trask, ancienne conservatrice du Musée de la Nouvelle-Écosse et spécialiste de cimetières, à collaborer avec moi en tant que photographe. Entre juin et septembre 2003, nous avons visité 61 cimetières situés dans 42

1. Jocelyn Saint-Arnaud, "La Mémoire du temps », dans Frontières (revue québécoise d'information, de recherche et de transfert de connaissances en études sur la mort), vol. $7, n^{\circ} 3,1995$, p. 3. 
paroisses ou missions différentes ${ }^{2}$. Dans le présent article, j'examinerai quatre vieux cimetières emblématiques en les plaçant d'abord dans un contexte historique, puis en regardant de près quelques-unes des pierres particulièrement intéressantes.

Sur sa carte de Port-Royal datée de 1686, Jean-Baptiste-Louis Franquelin montre l'église, une croix de chemin et le cimetière. Comme on le voit, le cimetière est entouré d'une clôture qui servait à délimiter la terre sacrée et à éloigner les animaux³. À l'intérieur du cimetière, il y a sept petites croix en bois et une autre croix plus grande (ill. 1). Il est important de souligner qu'il n'y a aucune preuve archéologique et aucune preuve écrite que les Français à Louisbourg ou que les Acadiens à PortRoyal, à Grand-Pré ou ailleurs, se soient servi de monuments de pierre pour marquer leurs tombes. À ce jour, les deux seules références que j'ai trouvées au sujet des monuments funéraires d'avant la déportation ne mentionnent que des croix en bois 4 .

Si les Acadiens marquaient leurs tombes, il semblerait donc que ce fût avec des croix en bois. Cela expliquerait pourquoi nous n'avons pas trouvé de stèles datant du xvIII ${ }^{\mathrm{e}}$ siècle, même si les premiers établissements acadiens après la déportation ont été fondés entre la fin des années 1760 et la fin des années 1780.

\section{Chéticamp}

La côte ouest de l'île du Cap-Breton se compose principalement de grès, roche sédimentaire friable et relativement facile à tailler. C'est sans doute à cause de l'abondance de cette pierre de sable que les plus

2. Pour les besoins de ce projet, j'ai défini un cimetière acadien comme un cimetière associé à une église catholique dans une communauté établie entièrement ou en grande partie par des Acadiens après 1764. J'ai reçu une subvention de recherche de 4000 dollars du Musée de la Nouvelle-Écosse, qui a couvert une partie des frais de déplacement $(6500 \mathrm{~km})$ et une partie du coût du développement des 2500 photos.

3. La carte de Franquelin offre le seul dessin connu d'une église acadienne du $x v \|^{\mathrm{e}}$ siècle. L'église en question a été détruite en 1690 par William Phips.

4. En expliquant comment les soldats de la Nouvelle-Angleterre avaient attaqué l'établissement micmac près de Port-Toulouse, à l'île Royale, l'abbé Pierre Maillard a écrit la phrase suivante, dans un rapport destiné aux autorités anglaises, An Account of the Customs and Manners of the Micmakis and Maricheets Savage Nations (S. Hooper \& A. Londres, Morley, 1758, p. 66) : "The same year, 1745, several bodies of the Savages, deceased, and buried at Port Tholouze [sic], were dug up again by the Bostoners, and thrown into the fire. The burying-place of the Savages was demolished, and all the crosses, planted on the graves, broke into a thousand pieces. "Dans le compte rendu de son expédition à pied vers 1790 dans la région de Tatamagouche, le pasteur presbytérien écossais note ce qu'il a vu : «traces also of a graveyard, with the cross still standing at the head of the graves ». Cf. George Patterson, Memoirs of the Rev. James MacGregor D.D., Philadelphia, Joseph M. Wilson, 1859, p. 263. 


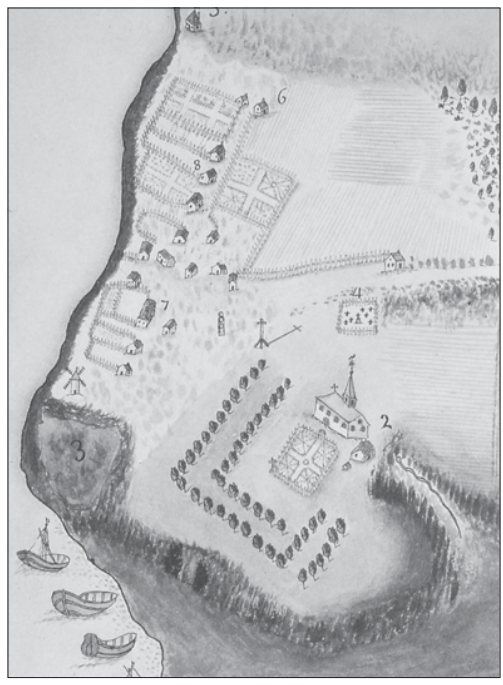

1. Port-Royal, carte de Jean-Baptiste-Louis Franquelin, 1686 (cliché, Bibliothèque nationale de France, Paris, département des cartes et des plans, SH groupe 132:2:2). Photographie de Sally Ross, 2003.

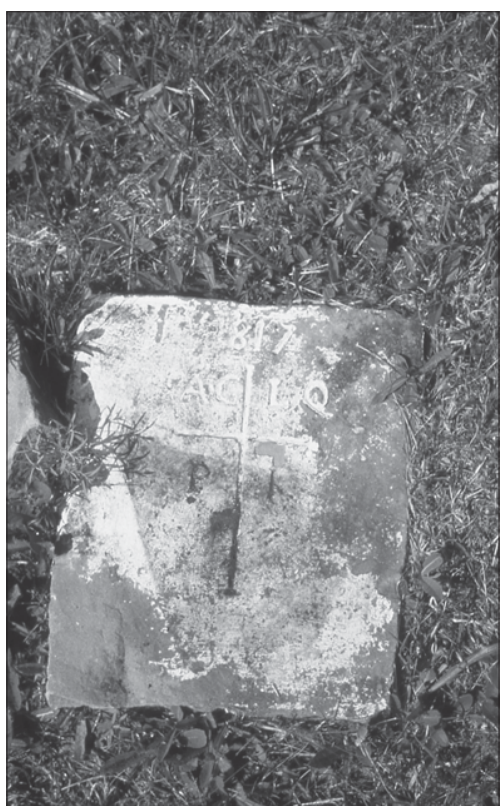

2. Chéticamp, pierre tombale portant l'inscription « ACLQPR 1817 ». Photographie de Sally Ross, 2003. 
vieux monuments funéraires acadiens de la Nouvelle-Écosse se trouvent à Chéticamp. Ce n'est sûrement pas par hasard que la grande région de Chéticamp ${ }^{5}$ (divisée maintenant en trois paroisses) est la seule région acadienne en Nouvelle-Écosse où le métier de tailleur de pierres tombales se soit développé ${ }^{6}$.

Le vieux cimetière de Chéticamp se trouve à l'intérieur des terres, tout près du Monument des Quatorze Vieux, qui a été érigé en 1955, à l'occasion du bicentenaire de la déportation, pour marquer l'emplacement de la deuxième église de Chéticamp et pour rendre hommage aux quatorze pionniers. Ce cimetière a servi du début des années 1800 jusqu'en $1868^{7}$. Après la création d'un autre cimetière beaucoup plus vaste en 1854 , le vieux cimetière a été abandonné, de sorte qu'il est retourné petit à petit à l'état sauvage. Voici ce que le père Anselme Chiasson m'a écrit en décembre 2003 : " J'avais fait un relevé de ce cimetière en 1936, mais incomplet à cause des broussailles impénétrables qui s'y trouvaient et qu'on a enlevées depuis. J'avais fait ce relevé avec mon oncle qui était né vers 1870 et [j'] avais noté des tombes de quelques pionniers de Chéticamp. ${ }^{8}$

En fait, comme l'a expliqué le père Charles Aucoin dans le Bulletin d'histoire et de généalogie de la Société Saint-Pierre, le vieux cimetière a été nettoyé dans les années 1950 sur l'initiative du père Jules Comeau, natif de Saulnierville et premier curé eudiste à servir à Chéticamp ${ }^{9}$. Après que les arbres et les broussailles ont été enlevés, les pierres tombales ont été replacées dans six rangées. Vers 1981, le père Donald Parent, eudiste

5. Les familles acadiennes ont colonisé la région de Chéticamp dans les années 1780 , une vingtaine d'années après l'établissement de Clare et d'Argyle. Pour une synthèse de l'histoire des régions acadiennes, voir Sally Ross et Alphonse Deveau, Les Acadiens de la Nouvelle-Écosse, Éditions d'Acadie, 1995, p. 110-198.

6. Dans son livre Chéticamp - Mémoires (réécrit et annoté par le père Anselme Chiasson), Anselme Boudreau mentionne les noms de trois tailleurs de pierres tombales (p. 111). Charlie Dan Roach a corrigé le nom des hommes et m'a fourni leurs dates : Alexandre (dit Bandet) Roach a vécu du 10 juin 1823 au 22 août 1887, P’tit Bélone Deveau a vécu du 25 septembre 1844 au 27 mars 1915 et Léopold (dit Léon à Raymond) Poirier est né le 9 novembre 1882 et décédé le 9 mars 1953.

7. Anselme Chiasson, Chéticamp - Histoire et traditions acadiennes, Moncton, Éditions des Aboiteaux, 1961, p. 118.

8. Lettre du père Anselme Chiasson capucin à Sally Ross datée du 17 décembre 2003.

9. Charles Aucoin, eudiste, "Ce qui reste de notre vieux cimetière », Bulletin d'histoire et de généalogie de la Société Saint-Pierre, vol. V, n 4, décembre 1988, p. 3-5. Les eudistes ont pris la charge de la paroisse de Chéticamp en 1953. Le père Aucoin indique que c'est à partir de cette époque que "l'entretien des cimetières est porté aux frais de la caisse paroissiale moyennant une collecte spéciale "pour le cimetière" ". Pour quelques notes biographiques sur le père Jules Comeau, voir Anselme Chiasson, Chéticamp - Histoire et traditions acadiennes, p. 153-154. Le père Jules Comeau (1899-1983) est enterré avec son frère, le père Camille Comeau (1895-1975), au cimetière Sainte-Marie à la Pointe-de-l’Église. 
du Québec en visite à Chéticamp pour quelques jours, a fait un relevé des inscriptions sur les 84 pierres tombales qui restaient. Le père Charles Aucoin a publié ce relevé ${ }^{10}$ et, à l'aide des notes d'Anselme Boudreau ${ }^{11}$, il a réussi à identifier un certain nombre des gens qui figurent dans les inscriptions. En 1991, il a publié son travail de défrichage en concluant son article de la façon suivante :

Soyons reconnaissants envers le Père Jules Comeau, le dévoué curé de Chéticamp de 1953 à 1964, d'avoir permis à ses paroissiens de redécouvrir le peu qui restait du Vieux Cimetière. Tout ravagé qu'il se présente aujourd'hui, nous nous devons d'en conserver avec le plus profond des respects les quelques lambeaux qui ont pu résister aux éléments et à l'insouciance blâmable des responsables. ${ }^{12}$

Lors de notre visite en juillet 2003, il ne restait plus qu'une soixantaine de pierres tombales plus ou moins intactes. Elles sont toutes en grès et, selon la tradition locale, elles ont été enduites d'une couche de peinture ou de chaux blanche. Certaines des stèles sont de forme rectangulaire et ne comprennent qu'une simple croix et des lettres ciselées de façon assez primitive. Quelques pierres sont datées de 1817. C'est la date la plus ancienne, mais il se peut que les deux ou trois pierres sans date soient plus anciennes. Un petit bloc couché porte l'inscription mystérieuse «ACLQ PR 1817 » (ill. 2). Sur la pierre de Basile Chiasson ${ }^{13}$, on voit une croix placée sur un triangle (iconographie que l'on retrouve sur d'autres pierres dans ce vieux cimetière) et l'inscription " BAC 1819 " (ill. 3). La pierre portant les initiales «P.O.Q » est sans doute celle de Peter O'Quin (Pierre Aucoin), qui est mort en 1863, à l'âge de 67 ans. L'inscription rudimentaire contraste avec la croix et l'ornementation ciselées par un sculpteur professionnel (ill. 4).

\section{Arichat}

En descendant la côte de l'île du Cap-Breton, on arrive au comté de Richmond, qui comprend aujourd'hui sept paroisses acadiennes. Celle d'Arichat est la plus ancienne, non seulement de l'île du Cap-Breton, mais

10. Charles Aucoin, op. cit., p. 3-5.

11. Anselme Boudreau (1890-1991) était un oncle du père Anselme Chiasson et auteur de Chéticamp : Mémoires, Les Éditions des Aboiteaux, 1996.

12. Charles Aucoin, eudiste, Bulletin d'histoire et de généalogie de la Société Saint-Pierre, vol. VIII, $\mathrm{n}^{\circ}$ 3, septembre 1991, p. 9.

13. Citant le registre, le père Aucoin dit que la pierre «D6 » est celle de Basile Chiasson, fils de Paul Chiasson et de Louise Boudreau, qui est décédé le 21 mars 1819 âgé d'environ 60 ans. 


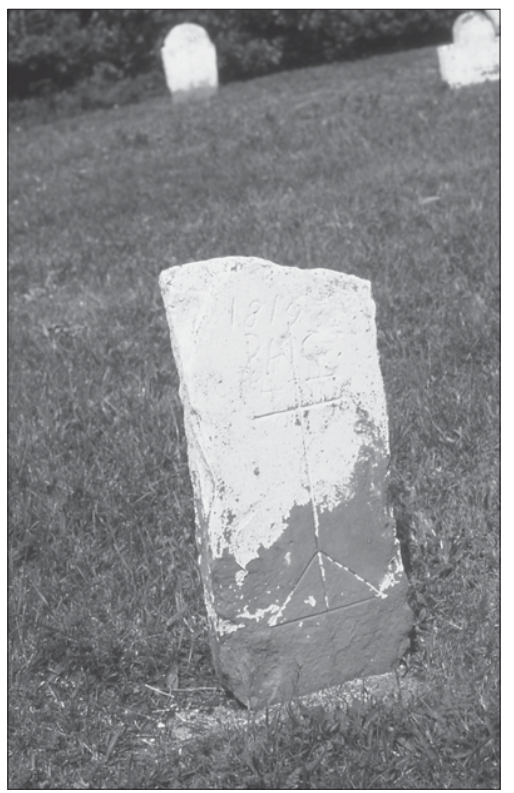

3. Chéticamp, pierre tombale de Basile Chiasson, 1819. Photographie de Sally Ross, 2003.

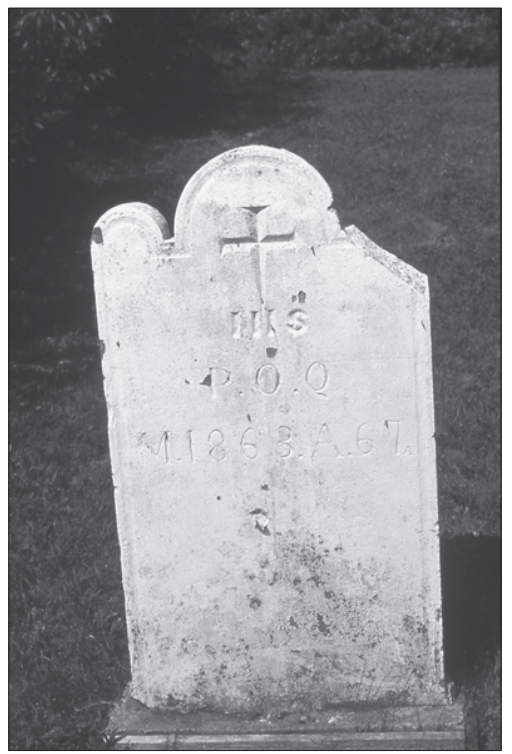

4. Chéticamp, pierre tombale de Pierre Aucoin, 1863. Photographie de Sally Ross, 2003. 
de tout le diocèse d'Antigonish. Établi vers 1765, Arichat est vite devenu un port de pêche extrêmement prospère, dont l'importance a éclipsé celle d'Halifax pendant un certain temps. Le vieux cimetière de l'église NotreDame-de-l'Assomption renferme quelques très beaux monuments sculptés en grès et en marbre qui reflètent cette prospérité d'autrefois. Même s'il ne semble pas avoir subi de gros travaux de nivellement, ce vieux cimetière a beaucoup changé au cours des années. Lorsque le généalogiste Stephen White l'a visité pour la première fois en 1967, la plupart des pierres étaient cachées par une masse impénétrable de mûriers sauvages ${ }^{14}$. Aujourd'hui, il y a une section en arrière qui n'est pas entretenue et où l'on peut voir quelques croix de bois et monuments en ciment datant des années 1920. Au début du xIx siècle, un certain nombre de familles irlandaises se sont établies à Arichat; c'est pourquoi on voit un mélange de noms irlandais et acadiens dans ce vieux cimetière.

En juillet 1812, Monseigneur Joseph-Octave Plessis, le onzième évêque de Québec, a fait une visite pastorale dans les provinces Maritimes. Quelques phrases dans son journal soulignent l'opulence relative d'Arichat : "C'est sur ce havre, qui a bien 3/4 de lieues de profondeur, qu'est située l'église Notre-Dame avoisinée par un élégant presbytère et par un vaste cimetière bien clos. Cette église [...] est, du reste, pourvue d'ornements, d'un clocher, de deux cloches, chose rare dans ces quartiers. ${ }^{15}$

À divers endroits au cours de son voyage, $M^{\mathrm{gr}}$ Plessis avait critiqué sévèrement les fidèles qui n'avaient pas encore clôturé leur cimetière. Par conséquent, en arrivant à Arichat, il a été très content de voir que le cimetière Notre-Dame était "bien clos », manifestation évidente du respect des paroissiens envers la terre sacrée et leurs défunts. L'église dont parle $M^{\text {gr }}$ Plessis a été remplacée en 1837 par l'église que l'on voit aujourd'hui ${ }^{16}$.

Les plus vieilles pierres tombales au vieux cimetière Notre-Dame datent des années 1840; elles n'existaient donc pas à l'époque de la visite de $M^{\text {gr }}$ Plessis. Cela veut dire qu'il n'y a aucun souvenir visible de

14. Stephen White a eu la gentillesse de me montrer les photos qu'il a prises à Arichat et à Arichat-Ouest en 1967 et 1971.

15. "Le Journal des visites pastorales de $M^{\text {gr }}$ Joseph-Octave Plessis en Acadie ", Les Cahiers de la Société historique acadienne, vol. 11, nos 1-2-3, 1980, p. 105.

16. En 1838, un incendie a détruit l'" élégant presbytère ", ainsi que tous les registres paroissiaux. Pour des détails sur la construction de la deuxième église, voir $A$. A. Johnston, A History of the Catholic Church in Eastern Nova Scotia, Antigonish, Saint Francis Xavier University Press, vol. II, 1960, p. 102. Le père Louis-Romuald Fournier, mort le 10 avril 1870 à Arichat-Ouest, est enterré à l'église Notre-Dame. Contrairement au Québec, l'inhumation dans les églises étaient extrêmement rare en NouvelleÉcosse; seuls quelques prêtres ont bénéficié de ce privilège. 
deux générations d'Acadiens enterrés à Arichat - phénomène dû, sans doute, à l'utilisation des croix en bois et qui caractérise tous les vieux cimetières acadiens de la Nouvelle-Écosse, à l'exception de celui de Chéticamp. D'ailleurs, la plus ancienne pierre tombale dans tout le comté de Richmond se trouve non loin d'Arichat, dans le vieux cimetière SaintHyacinthe à D'Escousse. II s'agit de la pierre érigée à la mémoire de Bélony Landry, décédé en 1835 à l'âge de 72 ans. Cette pierre est un simple bloc rectangulaire en grès, mais les monuments de grès au vieux cimetière d'Arichat sont d'un style beaucoup plus élégant.

Un des plus beaux monuments en grès à Arichat est dédié à la mémoire de deux prêtres : Augustus C. Lairez ${ }^{17}$, originaire de France, et Jean-Baptiste Maranda, originaire de l'île d'Orléans, qui sont décédés en 1834 et 1847 respectivement (ill. 5). L'abbé Maranda a dirigé la construction de la deuxième église Notre-Dame. Comme l'explique l'inscription, cette pierre a été érigée par les paroissiens d'Arichat et de D'Escousse. Au bas du monument, on trouve le nom du sculpteur «E. Fearon ». D’après Terry Punch, spécialiste de la généalogie irlandaise, "Fearon » est un nom typique de la région de Cork en Irlande. En toute probabilité, cette pierre a été importée. Cela expliquerait peut-être pourquoi l'inscription sur la tombe de deux prêtres francophones est en anglais. D'un style beaucoup plus modeste, la pierre tombale de Bregitte LeBlanc est aussi en grès (ill. 6). Elle comprend une croix et une belle draperie funéraire et porte l'inscription suivante : "Ici repose le corps de Bregitte LeBlanc, femme de Remi Forest décédé [sic] le 2nd [sic] sept 1849 à l'âge de 25 ans Requiescant [sic] in pace. Amen ». Selon la vieille tradition française, la femme est identifiée par son nom de jeune fille - coutume qui a été conservée beaucoup plus au Cap-Breton que dans le sud-ouest de la Nouvelle-Écosse. Une belle colonne de grès surmontée d'une croix en fer forgé est dédiée à la mémoire d'Honoré Martell, « né 3 d'août 1806 décédé 22 déc 1877 » (ill. 7). Premier Acadien du Cap-Breton à se faire élire à la Législature provinciale, Honoré Martell a été député de 1840 à 1863.

Les élégantes pierres tombales en grès constituent l'aspect le plus unique du vieux cimetière d'Arichat, mais il y a aussi une quinzaine de vieilles pierres très intéressantes en marbre ${ }^{18}$. On y voit, par exemple, le beau monument érigé à la mémoire de Brigitte LeBlanc, épouse d’Édouard LeBlanc et fille de Frédéric et Mariette Pothier, qui est décédée en 1861 à

17. Son nom s'écrit Lairez et non Lairey, comme l'indique l'inscription. Voir A.A. Johnston, op.cit., vol. II, p. 96.

18. Le marbre était utilisé en Nouvelle-Écosse entre 1845 et 1920 environ. Il était importé, puis sculpté par une des nombreuses compagnies de tailleurs de marbre dans la province. Pour plus de renseignements, voir Deborah Trask, Gravestone Carving and Carvers in Nova Scotia, Halifax, Nova Scotia Museum, 1978, p. 38, p. 83-92. 


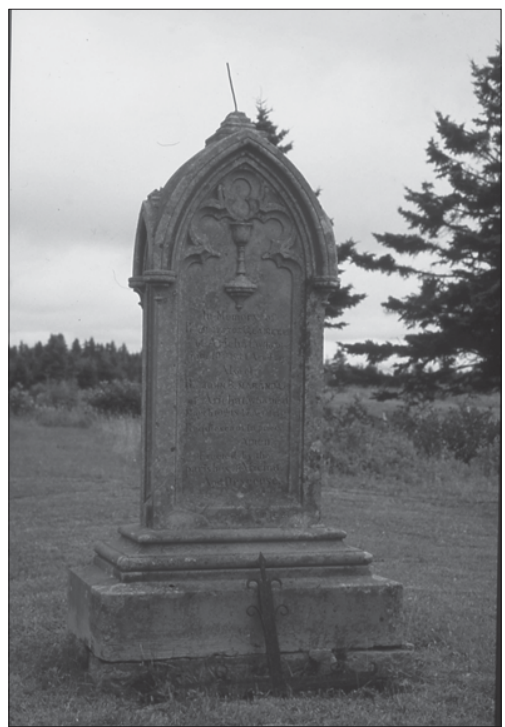

5. Arichat, monument dédié à la mémoire des pères Augustus C. Lairez et Jean-Baptiste Maranda, 1834 et 1847. Photographie de Sally Ross, 2003.

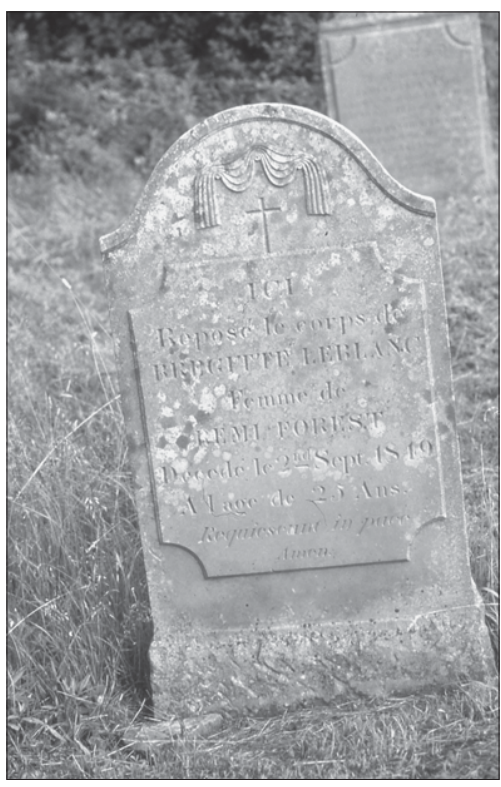

6. Arichat, pierre tombale de Bregitte LeBlanc, femme de Rémi Forest, 1849. Photographie de Sally Ross, 2003. 


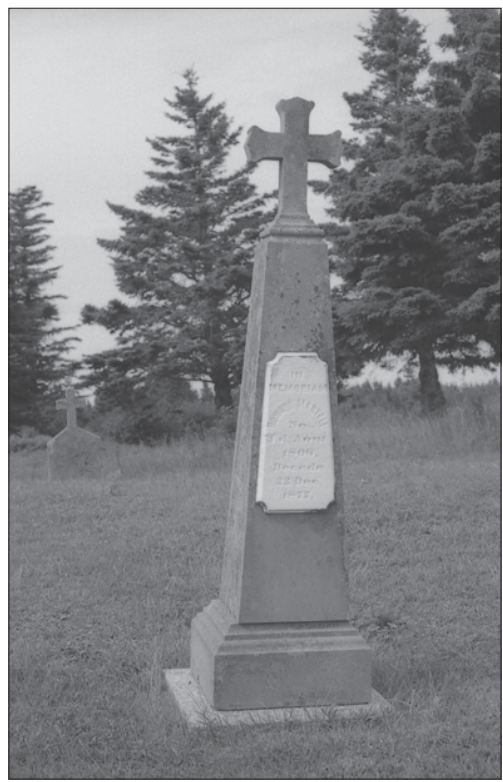

7. Arichat, pierre tombale d'Honoré Martell, 1877. Photographie de Sally Ross, 2003.

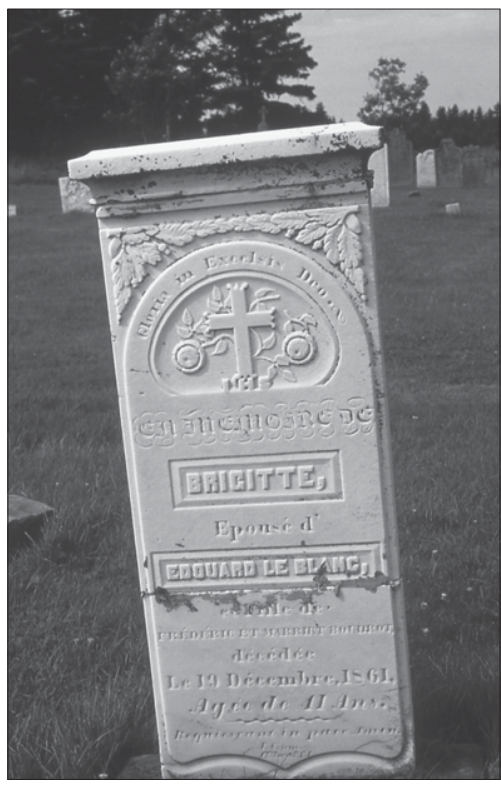

8. Arichat, pierre tombale de Brigitte LeBlanc, 1861. Photographie de Sally Ross, 2003. 
l'âge de 41 ans (ill. 8). Dans ce cas, la femme est identifiée non seulement en fonction de son mari, mais aussi de ses parents. Une inscription au bas du monument indique qu’il a été sculpté à Charlottetown.

\section{Pointe-de-l’Église}

Lors de sa visite à la baie Sainte-Marie en 1769, l'abbé CharlesFrançois Bailly a béni un terrain à la Pointe-à-Major qui avait déjà servi de lieu de sépulture en 1755. Ce premier cimetière de la baie Sainte-Marie a aussi servi de 1771 à 1791 . Un autre cimetière a été établi à côté de la petite église construite en 1786 sur la pointe qui a donné son nom au village de Pointe-de-l'Église. Une photographie de la troisième église SainteMarie $^{19}$ montre un cimetière entouré d'une belle clôture, qui renferme quelques monuments en marbre (signes d'une paroisse avec des familles nanties) qui subsistent encore aujourd'hui et dont les plus anciens datent des années 1860 (ill. 9). À l'avant-plan, on voit le grand monument en marbre érigé à la mémoire de l'abbé Sigogne en 1890 et déplacé deux ans plus tard lors de la translation de ses restes au terrain du collège SainteAnne. Cette vieille église était située à l'emplacement du calvaire qui se trouve au milieu du cimetière actuel que l'on voit dans une photographie aérienne, prise vers la fin des années 1960 (ill. 10). On y voit la grande pierre des champs qui porte l'inscription "Par ce signe vous deviendrez victorieux. Ici repose le corps de Rosalie THIBODAU décédée le 23 avril $1851 »^{20}$ (ill. 11). On y voit aussi de nombreuses croix blanches qui ont disparu aujourd'hui.

À mon avis, la présence de dix-neuf pierres des champs est un des aspects les plus intéressants et les plus intrigants du cimetière SainteMarie $^{21}$. Pour être plus précise, je devrais dire des pierres en ardoise, parce que toutes ces pierres n'ont pas été ramassées dans des champs. Dans le cas des seize pierres ayant une inscription visible, les dates de décès varient entre 1832 et 1902, mais plusieurs détails indiquent que la plupart de ces pierres ont été érigées longtemps après le décès du défunt

19. Appelée " la grande église du père Sigogne ", elle a été construite en 1829 sur l'emplacement d'une deuxième église construite en 1807 et détruite par un incendie en 1820. Pour une chronologie précise des églises et des cimetières, voir l'ouvrage Église Sainte-Marie 1905-2005, Pointe-de-l'Église, Conseil paroissial de la paroisse Sainte-Marie, 2005, p. 107-110 et 118.

20. On voit ces croix blanches dans une photo de cette grande pierre des champs prise par Jean-Claude Dupont, en 1976, et publiée dans son livre Histoire populaire de l'Acadie, aux Éditions Leméac, 1979, p. 136.

21. Il y a une pierre des champs au cimetière de la Pointe-à-Major. La pierre en question est celle de Marie Doucet, décédée en 1771, mais je crois qu'elle a été placée lors de l'aménagement du cimetière par la Société historique de la baie Sainte-Marie en 1981. Il y a aussi une petite pierre des champs à Doucetteville datée de 1921. 


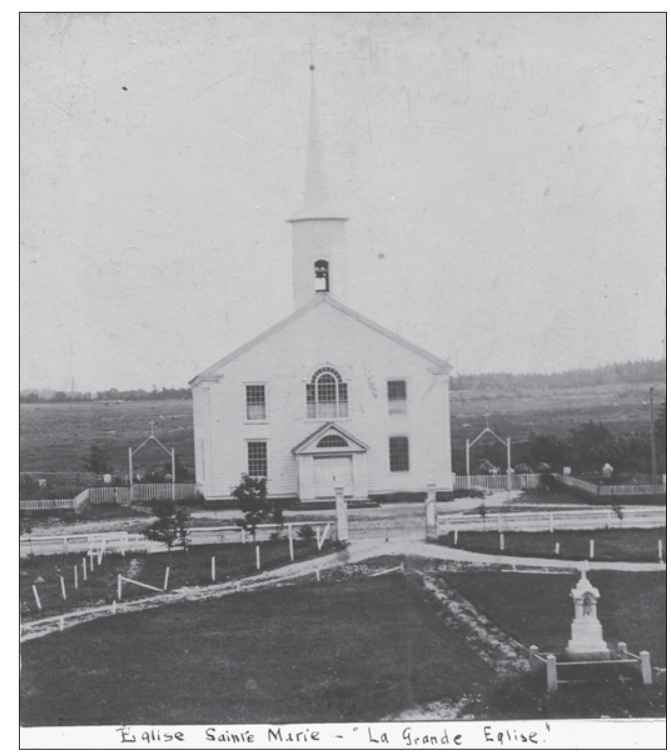

9. Pointe-de-l'Église. La « grande église » de l'abbé Sigogne, érigée de 1820 à 1829. Photographie du Centre acadien.

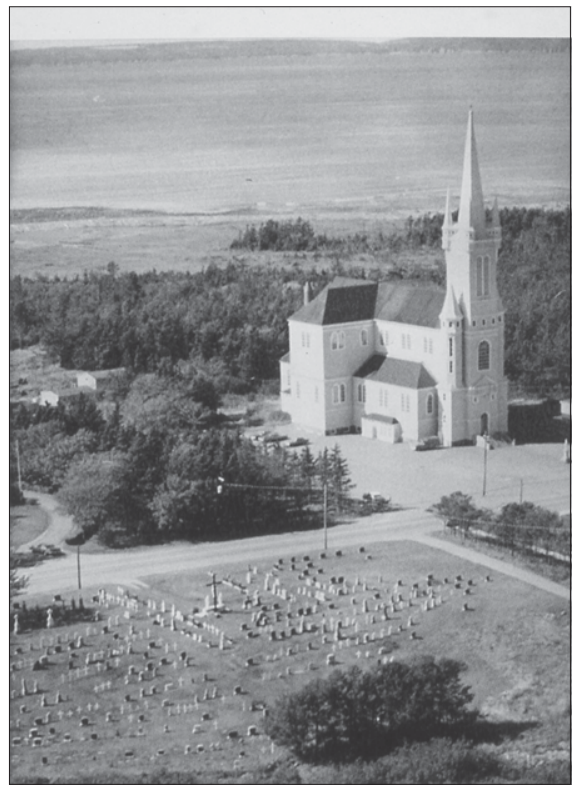

10. Pointe-de-l'Église, église et cimetière Sainte-Marie, vers la fin des années 1960. Photographie de Sally Ross, 2003. 


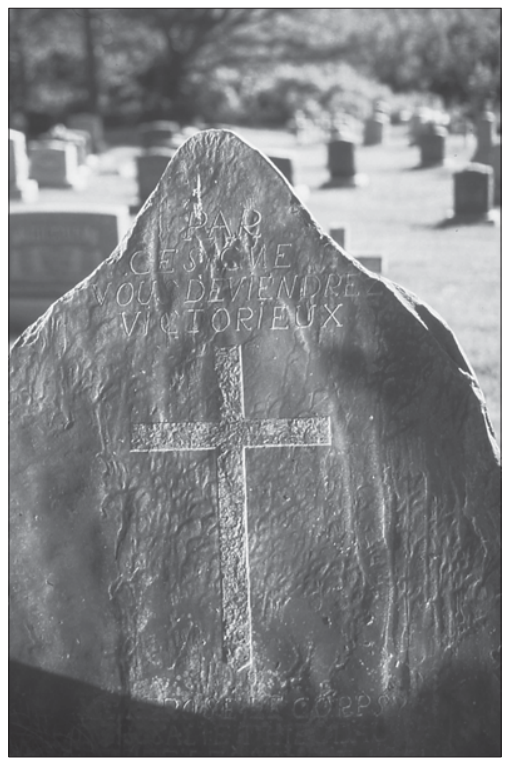

11. Pointe-de-l'Église, pierre tombale de Rosalie Thibodau, 1851. Photographie de Sally Ross, 2003.

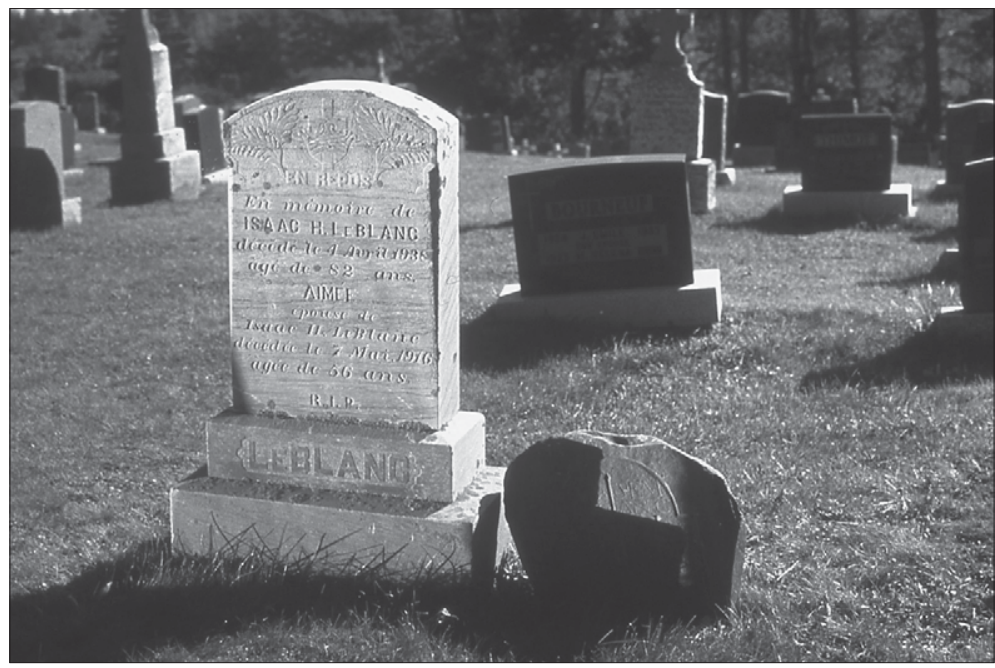

12. Pointe-de-l'Église, pierre tombale d'Isaac et Aimée LeBlanc, 1938 et 1916. Photographie de Sally Ross, 2003. 
ou de la défunte. Il va sans dire que cela n'enlève rien à leur importance comme témoins du passé. Certaines pierres ont été sculptées de façon artisanale, alors que d'autres sont l'œuvre d'un tailleur professionnel. Quelques pierres comportent des formulations et une iconographie que l'on ne trouve dans aucun autre cimetière acadien en Nouvelle-Écosse - ce qui n'est pas surprenant étant donné la proximité du collège et l'influence des eudistes nés en France.

À côté du monument en marbre dédié à la mémoire d'Isaac et d'Aimée LeBlanc (ill. 12), on voit une petite pierre des champs ${ }^{22}$ qui comporte une croix placée dans un cadre romain (iconographie que l'on ne trouve pas ailleurs en Nouvelle-Écosse) et l'inscription suivante : «ASSEM LEBLANC D C D E L 1843 agee $75 \mathrm{~N}$ » (ill. 13).

L'inscription sur la plus grande des pierres des champs que l'on trouve au cimetière Sainte-Marie se lit comme suit : "Ici repose le corps de Joseph Dugas décédé le 29 octobre 1858 âgé de 92 ans / Il était le premier Acadien né à Clare / Requiescat in pace » (ill. 14). Dans son livre Les personnes éminentes, l'historien Alphonse Deveau indique qu'il y a une erreur dans l'inscription parce "qu'on a toujours cru faussement que Joseph Dugas, fils est né en 1766 tandis que le registre de l'abbé Bailly établit clairement qu'il est né en $1768 »^{23}$. On peut conclure d'après cette note d'Alphonse Deveau que cette pierre n'a pas été placée à l'époque de la mort de Joseph Dugas.

La pierre de Charlotte Deveau et Marc Sonier vient sans doute d'une carrière d'ardoise et semble être l'œuvre d'un tailleur professionnel. Située en haut de la pierre, pour laisser la place à deux grandes croix, l'inscription comprend le nom des défunts et la date de décès (1855) écrite en toutes lettres (ill. 15). Nous n'avons vu aucun autre exemple en Nouvelle-Écosse d'une date écrite de cette façon.

De style artisanal, la pierre des champs ayant les dates de décès les plus récentes est décorée de deux petites croix au-dessus de deux inscriptions placées côte à côte. L'inscription se lit : "Catherine DOUCET décédé janvier 91899 age 65 / Alban DOUCET décédé mai 211902 age 86 » (ill. 16).

\section{Pubnico-Ouest}

Dans son Histoire religieuse de Pubnico et dans un dépliant publié en 1981 à l'occasion de la dédicace du monument commémoratif²4 érigé au

22. Jean-Claude Dupont en a publié une photo prise en 1976 dans son livre Histoire populaire de l'Acadie, p. 136.

23. J. Alphonse Deveau, Les personnes éminentes, Les Éditions Lescarbot, 1988, p. 94.

24. Le monument en ciment, qui comprend une plaque historique, est surmonté de la croix du clocher de la deuxième église. Le projet a été subventionné par le programme 


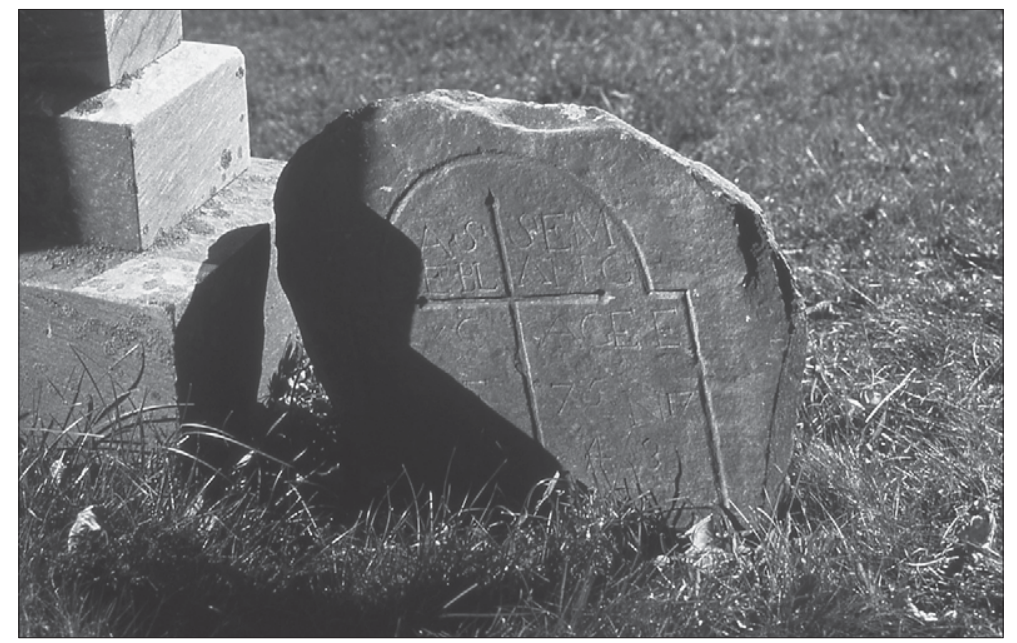

13. Pointe-de-l'Église, pierre portant l'inscription «ASSEM LEBLANC D C D E L 1843 agee 75 N ». Photographie de Sally Ross, 2003.

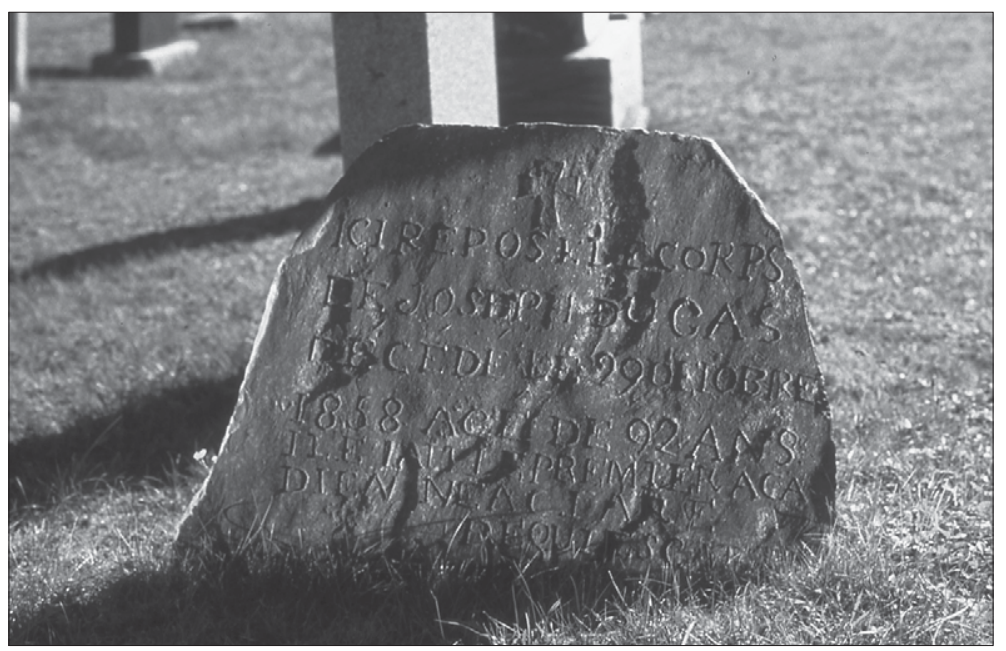

14. Pointe-de-l'Église, pierre des champs dédiée à la mémoire de Joseph Dugas, premier Acadien né à Clare. Photographie de Sally Ross, 2003. 


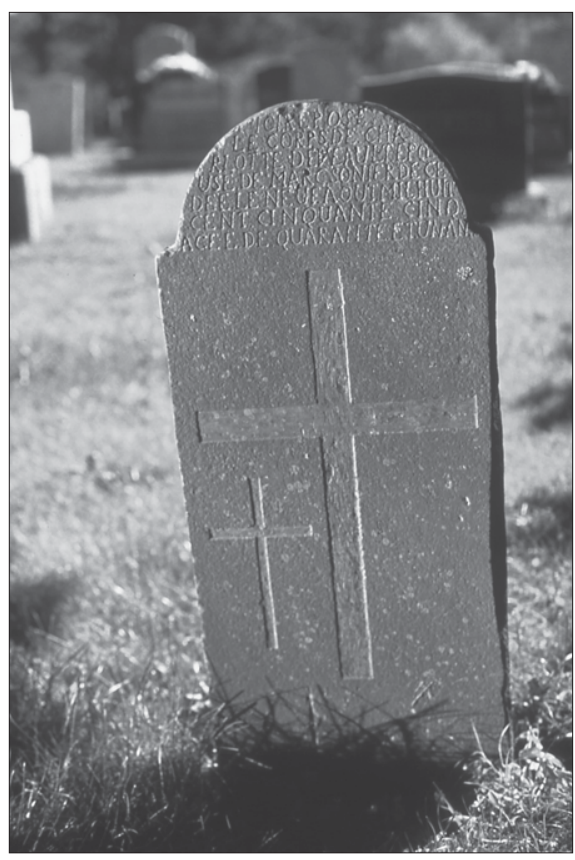

15. Pointe-de-l'Église, pierre tombale de Charlotte Deveau et Marc Sonier, 1855. Photographie de Sally Ross, 2003.

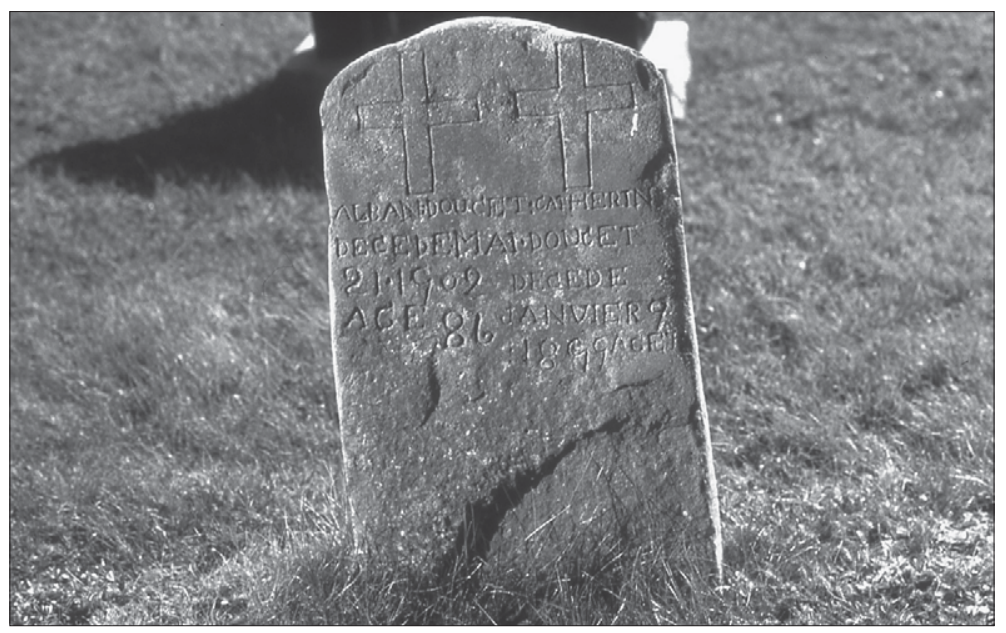

16. Pointe-de-l'Église, pierre tombale de Catherine et Alban Doucet, 1899 et 1902. Photographie de Sally Ross, 2003. 
vieux cimetière de Pubnico-Ouest-le-Bas, le père Clarence d'Entremont nous donne beaucoup de renseignements sur les deux cimetières qui ont précédé celui de l'église Saint-Pierre actuelle. Dans une photographie de la deuxième église ${ }^{25}$ qui a servi de 1853 à 1901 (ill. 17), on peut déceler quelques monuments en marbre, dont le plus ancien est celui de Frances Amiro (jeune épouse de Louis R. Amiro), décédée en 1854, date à laquelle les premières pierres tombales en marbre apparaissent dans les cimetières acadiens d'Argyle ${ }^{26}$ (ill. 18). Le monument de Frances Amiro est d'autant plus intéressant que l'on y trouve le nom du tailleur de marbre, John D. Drake, d'Halifax ${ }^{27}$. Quelques-uns des autres monuments au vieux cimetière portent des inscriptions en français. On remarque aussi des pierres avec des noms irlandais, parce que la petite communauté de Pubnico Head (Tête-de-la-Rivière) n'avait pas de cimetière catholique à l'époque. Les vieilles pierres en marbre ont été déplacées à deux reprises lors des travaux de terrassement dans les années 1950, mais, comme c'était souvent le cas, elles n'ont pas été remises à l'emplacement des tombes. S'il y a eu des croix ou autres monuments en bois, on ne les a pas conservés.

La première inhumation dans le grand cimetière qui se trouve derrière l'église Saint-Pierre à Pubnico-Ouest a eu lieu le 28 octobre $1892^{28}$. Il s'agissait de l'enterrement d'Actime d'Entremont, mais à ma connaissance aucun monument ne marque sa tombe. Le grand cimetière Saint-Pierre est remarquable surtout à cause de la grande variété des monuments. On y trouve toutes sortes de matériaux : bois, marbre, zinc, fer, ciment et granit. Il y a un nombre très impressionnant de grandes colonnes en marbre

«Nouveaux Horizons » à l'occasion du $100^{\mathrm{e}}$ anniversaire du choix de l'Assomption comme fête nationale des Acadiens.

25. Le premier cimetière était situé de l'autre côté du chemin et semble avoir servi de 1810 jusqu'à la bénédiction du deuxième cimetière par $M^{\text {gr }}$ Walsh, archevêque d'Halifax, en 1853. Le dernier enterrement, celui de Marie-Jeanne d'Entremont, a eu lieu le 6 juillet 1901. La démolition de la deuxième église (construite en 1840) a commencé en 1902 et s'est terminée en 1903, douze ans après la célébration de la première messe dans l'église actuelle. Voir Clarence-J. d'Entremont, Histoire religieuse de Pubnico, Les Éditions Lescarbot, 1992, p. 93-94 et p. 149.

26. En fait, il s'agit de deux autres cimetières seulement. La pierre d'Ambroise Potier, décédé aussi en 1854, est la plus ancienne du vieux cimetière de Sainte-Anne-duRuisseau et la pierre du père Joannis Nugent est la plus ancienne de Wedgeport. Dans son Histoire de Wedgeport (p. 70 de la nouvelle édition de 1995), le père Clarence d’Entremont indique que la pierre tombale du père Nugent a été érigée en 1854, deux ans après sa mort.

27. Pour une annonce publicitaire de la compagnie Drake, voir Deborah Trask, op. cit., p. 85 .

28. Clarence-J. d’Entremont, Histoire religieuse de Pubnico, Les Éditions Lescarbot, 1992, p. 142. 


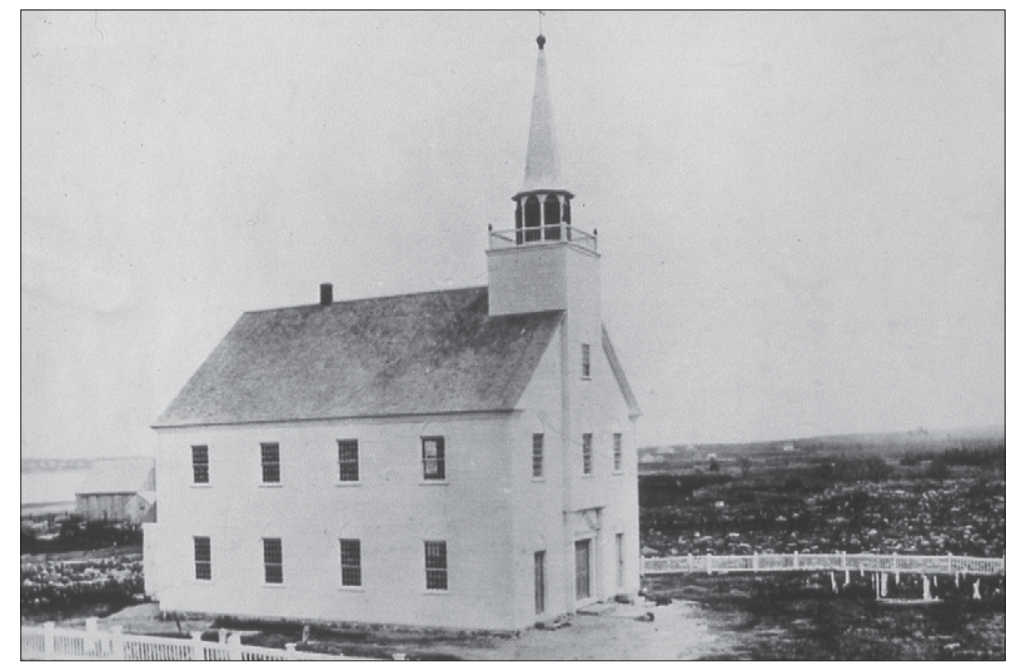

17. Pubnico-Ouest-le-Bas, vieille église vers 1900. Photographie de Sally Ross, 2003.

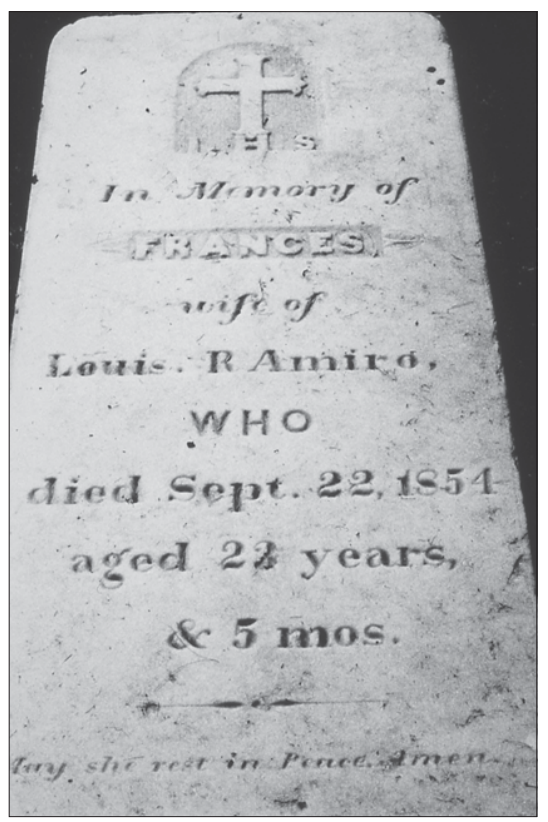

18. Pubnico-Ouest-le-Bas, pierre tombale de Frances Amiro, 1854. Photographie de Sally Ross, 2003. 
gris typiques des années 1920, comme celle (ill. 19) qui est dédiée à la mémoire de Jacques d'Entremont, perdu en mer en 1870 à l'âge de 37 ans, et de son épouse Agnès décédée en 1923, à l'âge de 82 ans. D'ailleurs, en se promenant dans les cimetières d'Argyle, on est frappé par le nombre d'hommes qui ont perdu leur vie en mer $^{29}$.

La grande croix en fonte moulée dédiée à la mémoire d’Adrien Amirault (décédé en 1909 à l'âge de 41 ans et 3 mois) est un des monuments les plus spectaculaires du cimetière Saint-Pierre (ill. 20). Cette croix décorée de fleurs-de-lys et de coquilles Saint-Jacques porte la marque de la fonderie A. Bélanger de Montmagny, Québec. D’après mes recherches, cette compagnie, fondée en 1867 , se spécialisait dans la fabrication des poêles à bois. Il est intéressant de noter que les croix en fonte de fer fabriquées par la compagnie Bélanger n'étaient plus permises dans les cimetières de Montmagny à partir de septembre 1921, parce qu'elles étaient fixées dans une base en ciment qui s'effritait trop vite, faisant tomber la croix ${ }^{30}$. Il faut croire que le ciment de Pubnico était de meilleure qualité que celui de Montmagny! Il reste à savoir comment ou pourquoi la famille Amirault a choisi de se procurer une de ces magnifiques croix ${ }^{31}$.

Le monument dédié à Lelian A. L. Amiro est tout à fait exceptionnel (ill. 21). Il s'agit d'une stèle faite d'une seule planche de bois ${ }^{32}$. On voit, d'après l'inscription en anglais, que Lelian Amiro est morte en 1897 à l'âge de 22 ans et 2 mois - six ans après la construction de l'église SaintPierre. De loin, le monument ressemble beaucoup à une pierre tombale en marbre, mais en le regardant de près on voit le grain du bois sous les vestiges d'une vieille couche de peinture blanche. C'est à dessein sans doute que le sculpteur voulait imiter l'apparence noble du marbre. L'inscription et la décoration (une croix en relief dans un cadre tréflé) sont ciselées dans le bois et peintes en noir. Les stèles en bois sont extrêmement rares maintenant en Nouvelle-Écosse. Évidemment, on ne peut pas affirmer avec certitude que celle-ci date de 1897, mais ce n'est pas impossible. Il y en a une autre presque identique datée de 1904 qui

29. En 2003, à l'occasion du $350^{\circ}$ anniversaire de Pubnico, un monument a été érigé à l'entrée du village à la mémoire des 45 hommes qui ont perdu leur vie en mer monument sans doute inspiré par le Gloucester Fisherman's Memorial, dévoilé en 1999, sur lequel on trouve les noms des 1200 pêcheurs de la Nouvelle-Écosse qui ont perdu leur vie à bord des bateaux provenant de Gloucester, Massachusetts.

30. " Les Cimetières de Montmagny ", 〈http://pages.globetrotter.net/st-thomas/ cimetiere.htm>, p. 10.

31. Il n'y a qu'une autre croix de ce genre en Nouvelle-Écosse, mais elle est plus petite et elle ne porte pas la marque du fabricant. Elle se trouve au cimetière Saint-JeanBaptiste à Rivière-Bourgeois au Cap-Breton.

32. Il se peut que ce soit du mélèze ("violon ») parce que cette essence est réputée pour sa durabilité et sa résistance à l’humidité. 


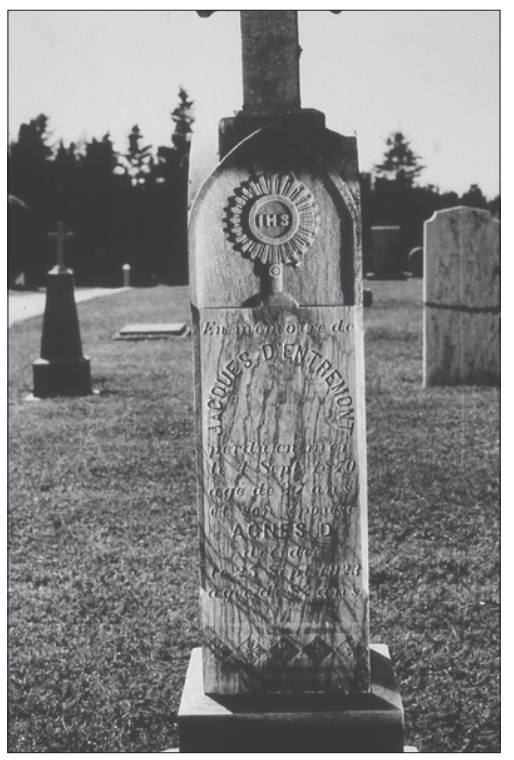

19. Pubnico-Ouest, monument érigé à la mémoire de Jacques d'Entremont perdu en mer en 1870. Photographie de Sally Ross, 2003.

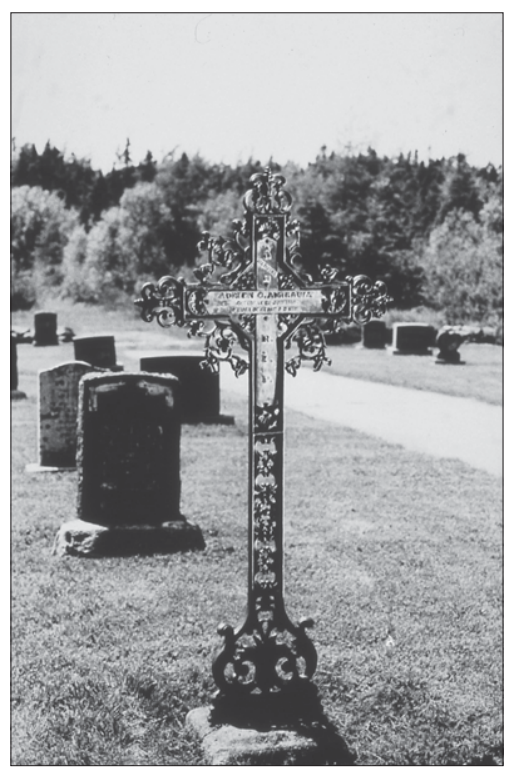

20. Pubnico-Ouest, croix en fonte moulée dédiée à la mémoire d'Adrien Amirault, 1909. Photographie de Sally Ross, 2003. 
se trouve dans la paroisse voisine de Saint-Michel à Wedgeport. D'après mes observations sur le terrain, les croix et les stèles en bois survivent très longtemps, à condition d'être faites d'une essence comme la pruche ou le mélèze, qui résiste à l'humidité, et à condition d'être renforcées par des tiges de métal au niveau du sol. Tous les monuments funéraires, quelle que soit leur origine, sont des témoins silencieux du passé, mais ces exemples de l'art populaire sont particulièrement émouvants.

\section{Conclusion}

Ces vieux cimetières dispersés aux quatre coins de la NouvelleÉcosse donnent une idée de la richesse du patrimoine funéraire acadien du $x_{1 x}{ }^{e}$ siècle. Même si les souvenirs visibles de la première génération d'Acadiens et d'Acadiennes enterrés après la déportation sont rarissimes, les monuments qui nous restent montrent les caractéristiques régionales, les différences sociales et une variété de façons de préserver la mémoire des bien-aimés disparus.

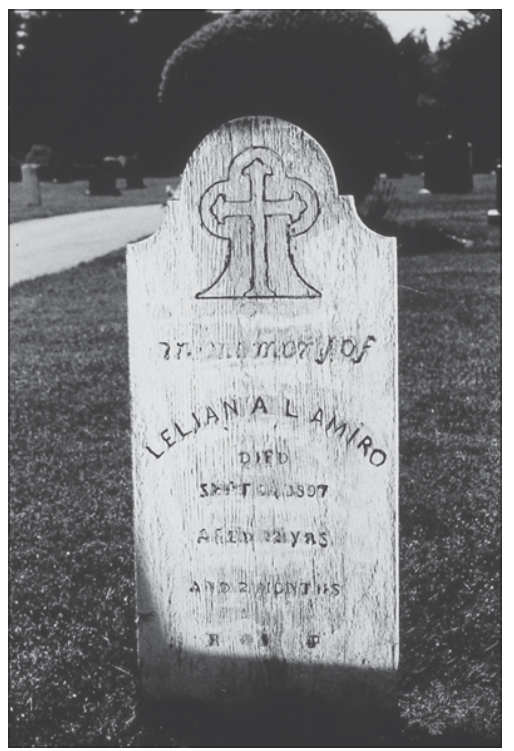

21. Pubnico-Ouest, monument en bois dédié à la mémoire de Lelian A. L. Amiro, 1897. Photographie de Sally Ross, 2003. 


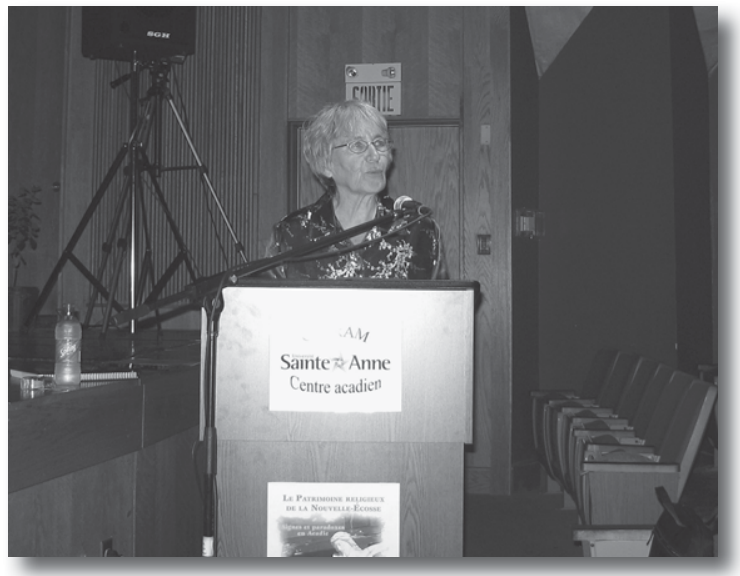

Sally Ross

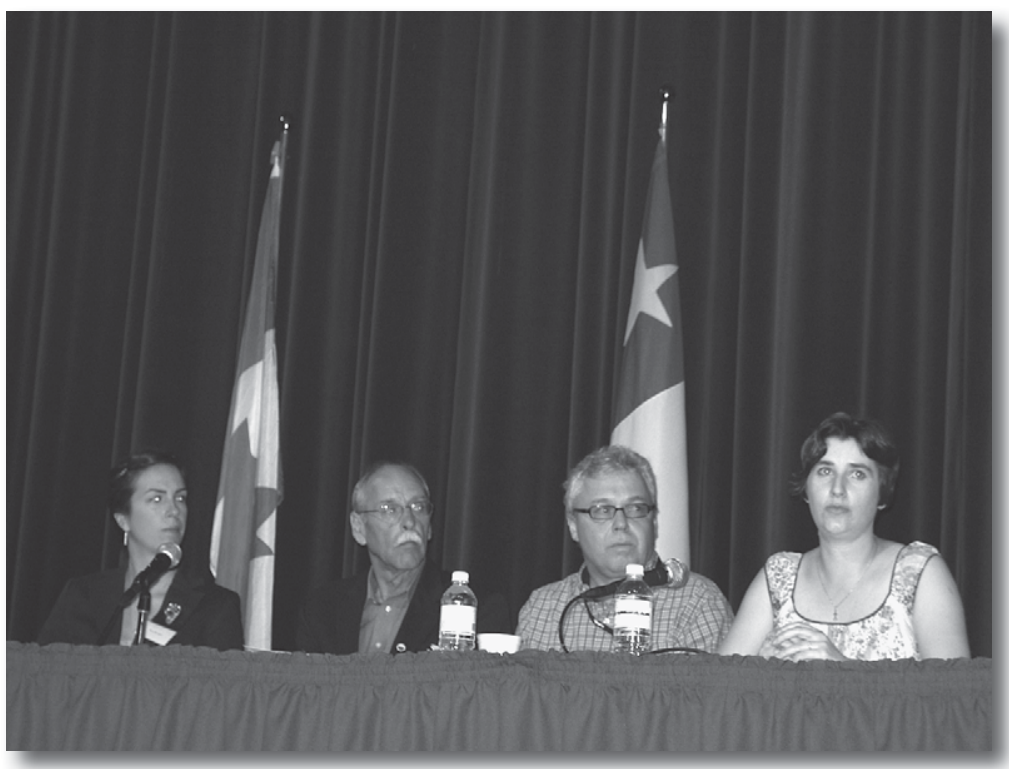

Jill Scott, Delphis Comeau, Marc Lavoie et Gwénaëlle Le Parlouër 